\title{
Antimicrobial susceptibility, virulence determinants profiles and molecular characteristics of Staphylococcus epidermidis isolates in Wenzhou, eastern China
}

Yinjuan Guo ${ }^{1 \dagger}$, Yu Ding ${ }^{2+}$, Li Liü ${ }^{3}$, Xiaofei Shen ${ }^{3}$, Zhihao Hao ${ }^{3}$, Jingjing Duan ${ }^{3}$, Ye $\mathrm{Jin}^{3}$, Zengqiang $\mathrm{Chen}^{3}$ and Fangyou $\mathrm{Yu}^{3^{*}}$ (i)

\begin{abstract}
Background: Staphylococcus epidermidis has emerged as an often encountered pathogen responsible for hospitalacquired infections. The aim of present study is to investigate the microbiological characteristic of $\mathrm{S}$. epidermidis isolates isolated from sterile specimens and skin in a Chinese tertiary hospital.

Methods: A total of 223 non-duplicate S. epidermidis were collected from various sterile specimens of inpatients among 10 years in Wenzhou, China. 106 S. epidermidis obtained from the skin (urethral orifices) of healthy volunteers. All isolates were tested for antimicrobial susceptibility. PCR was used to detect the virulence- and resistance-associated genes and 7 housekeeping genes to determine the sequence types (STs) of selected isolates.

Results: The resistance rates to antimicrobials tested except linezolid and vancomycin and the prevalence of methicillinresistant $S$. epidermidis (MRSE) of $S$. epidermidis clinical isolates were significantly higher than those among colonized isolates $(P<0.05)$. The positive rates of virulence-associated genes including aap, sesl, ACME-arCA, IS256, bhp, altE, aae and gehD for $S$. epidermidis clinical isolates were significantly higher than those for colonized isolate $(P<0.05)$. A total of 60 STs including 28 from clinical isolates and 32 from colonized isolates were identified by MLST. A novel, rarely encountered clone, ST466, was found to be the second prevalent clone among clinical isolates. The great majority of the S. epidermidis isolates tested (73.86\%) belonged to clone complex 2 (CC2). Compared with ST2, ST130, ST20 and ST59 clones, ST466 clone had the highest resistance rate to tetracycline (50.00\%), the second highest prevalence of ACME-arcA (65.00\%), bhp $(30.00 \%)$ and qacA B (65.00\%), very low prevalence of carriage of icaA $(0.00 \%)$ and biofilm formation $(0.00 \%)$, the lack of ses/ and high prevalence of aap, altE and aae (>90\%), which was similar to the characteristics of ST59 clone with one locus difference from ST466. ST466 clone competence with Staphylococcus aureus was relatively stronger, relative to ST2, ST20, ST130 and ST59 clones.
\end{abstract}

Conclusion: Taken together, a high-level of genetic diversity was found between clinical and colonized S. epidermidis isolates. A novel ST466 clone with distinct and similar characteristics relative to other prevalent clones, emerging as a prevalent clone in China, should be of major concern.

Keywords: Staphylococcus epidermidis, Resistance, Virulence-genes, MLST

\footnotetext{
* Correspondence: wzjxyfy@163.com

†Yinjuan Guo and Yu Ding contributed equally to this work.

${ }^{3}$ Department of Laboratory Medicine, the First Affiliated Hospital of Wenzhou

Medical University, Wenzhou 325000, China

Full list of author information is available at the end of the article
}

(c) The Author(s). 2019 Open Access This article is distributed under the terms of the Creative Commons Attribution 4.0 International License (http://creativecommons.org/licenses/by/4.0/), which permits unrestricted use, distribution, and reproduction in any medium, provided you give appropriate credit to the original author(s) and the source, provide a link to the Creative Commons license, and indicate if changes were made. The Creative Commons Public Domain Dedication waiver (http://creativecommons.org/publicdomain/zero/1.0/) applies to the data made available in this article, unless otherwise stated. 


\section{Background}

Staphylococcus epidermidis is a common commensal bacterium, which belongs to coagulase-negative Staphylococci (CoNS). It is now recognized as a relevant opportunistic pathogen and isolated prevalently from human epithelia and colonizes predominantly the axillae, head and nares [1]. However, in recent decades, as the S. epidermidis is one of the often encountered biofilm-producing bacteria, it has emerged as a common cause of hospitalacquired infections associated with the use of indwelling or implanted foreign bodies [1]. Biofilm formation is the most important factor for the establishment of $S$. epidermidis. The principal component of biofilms a polysaccharide intercellular adhesin (PIA) or polymeric $N$-acetyl-glucosamine (PNAG) produced by the four gene ica $A D B C$ operon [2]. In addition to PIA, many components have been found to be associated with biofilm formation, such as accumulationassociated protein (Aap), biofilm-associated protein (Bap) and IS256. A novel genomic island named arginine catabolic mobile element (ACME) may increase the colonized capacity of S. epidermidis to the human skin, mucosal surfaces and in-dwelling medical devices [3]. Furthermore, as for nosocomial pathogen, increasing rates of antibiotic resistance are an even greater problem for S. epidermidis limits our therapeutic options. In previous decades, a continuous decreased susceptibility of $S$. epidermidis to most of the clinically available antibiotics was found. Methicillin resistance in Staphylococci, which is associated with the presence of the mecA gene carried by a genetic mobile element called the staphylococcal chromosomal cassette mec (SCCmec), is the other very important factor in the establishment of $S$. epidermidis as a nosocomial pathogen [1, 4]. Molecular typing has become establish for the population analysis of nosocomial S. epidermidis strain and is a good method for addressing the lack information compared with that of $S$. aureus [5]. By using multilocus sequence typing (MLST), a typing method based on the sequence polymorphism of fragments of seven housekeeping genes, it was revealed several epidemiology clonal lineages disseminated worldwide [5]. Clonal complex 2 (CC2), the most predominant of these lineages, is composed of a large number of sequence types $[6,7]$. Limited information is available on the molecular epidemiology of S. epidermidis clinical isolates in China. In the present study, the aim is to investigate the antimicrobial resistance profiles, virulence determinants profiles and molecular characteristics of S. epidermidis isolates.

\section{Methods}

\section{Bacterial isolates}

\section{a) Clinical S. epidermidis isolates}

A total of 223 non-duplicate S. epidermidis isolates were isolated from various sterile specimens of inpatients from
2002 to 2008 and 2012 to 2014 at the bacterial library of the first Affiliated Hospital of Wenzhou Medical University, Wenzhou, eastern China. Each isolates were transferred to a blood plate and then cultured overnight in $5 \mathrm{ml}$ TSB at $37^{\circ} \mathrm{C}$ with shaking at $200 \mathrm{rpm}$ from glycerin broth stored in $-80^{\circ} \mathrm{C}$ of bacterial library. The S. epidermidis isolates from patients with clinical signs and symptoms of infection were considered for invasive isolates and included for investigation. The isolates included were isolated from blood (67 isolates), catheters (52 isolates), pus (48 isolates, 7.9\%) and other specimens (56 isolates).

\section{b) Colonized S. epidermidis isolates}

The definition of colonization is bacteria often fall from different environments to the human body, and can settle and grow in a certain part, and breed offspring. 106 colonized S. epidermidis isolates were isolated from the urethral orifices of healthy volunteers with no symptoms. There were $76 \%$ female and $24 \%$ male from 18 to 30 years old without any diseases. The isolates tested were identified as S. epidermidis by VITEK automatic microbiology analyzer (bioMe'rieux, Marcy l'Etoile, France).

\section{Antimicrobial susceptibility testing}

In vitro antimicrobial susceptibility testing was determined by the disk diffusion method with antimicrobials in accordance with the standards recommended by the Clinical and Laboratory Standards Institute(CLSI) [8], including penicillin $(10 \mu \mathrm{g})$, erythromycin $(15 \mu \mathrm{g})$, clindamycin $(2 \mu \mathrm{g})$, linezolid $(30 \mu \mathrm{g})$, tetracycline $(30 \mu \mathrm{g})$, trimethoprim/ sulfamethoxazole $(1.25 / 23.75 \mu \mathrm{g})$, gentamicin $(10 \mu \mathrm{g})$, ciprofloxacin $(5 \mu \mathrm{g})$, and levofloxacin $(5 \mu \mathrm{g})$. All disks were obtained from Oxoid Ltd. S. aueus ATCC 25923 was used as control strain for determining the antimicrobial susceptibility for S. epidermidis isolates in accordance with CLSI breakpoints. The minimum inhibitory concentration value of vancomycin was determined with the standard agar dilution method recommended by the CLSI [8].

\section{MLST typing and goeBURST algorithm}

Multilocus sequence typing of the $S$. epidermidis strains was determined by amplifying of the seven housekeeping genes including $\operatorname{arcC}$, aroE, gtr, mutS, pyrR, tpiA and $y q i L$ as described previously [9]. The fragments containing seven housekeeping genes were sent to TSINGKE Company (Hangzhou, China) for purified and DNA sequencing service. The numbers of alleles and sequence types were assigned using online database (http:// www.mlst.net). The goeBURST algorithm (http://goeBURST.phyloviz.net) was used to infer the evolutionary relatedness of the STs. 
PCR method for detection of virulence- and resistanceassociated genes

All isolates included were tested for the presence of the $m e c A$ gene by PCR amplification using the primers described previously [10]. The presence of the virulenceassociated genes including icaA, sesI, aap, arcA, IS256, altE, bhp, aae and gehD was determined by PCR using primers described previously [7, 11-15]. PCR was used to detect whether the isolates tested harbored mupirocin-resistance protein-encoding gene $(m u p A)$ and chlorhexidine-based antiseptic resistance loci $(q a c A / B)$ according to previously described primers [16].

\section{Biofilm formation assay}

The biofilm formation assay was performed as described by O'Neill et al. using the flat bottom 96-wellpolystyrene plates (BD Biosciences) [17]. In brief, each strain grew overnight in trypticase soy broth (TSB) (BD, USA). Then, the bacterial suspensions $\left(1 \times 10^{8} \mathrm{CFU} / \mathrm{ml}\right)$ were diluted 1:200 in fresh TSB. Wells of a 96-well microplate were then inoculated with two hundred microliter bacterial suspensions. TSB served as background controls. After $24 \mathrm{~h}$ incubation at $37^{\circ} \mathrm{C}$, the walls were removed and washed three times with $200 \mu \mathrm{l}$ of PBS. Then the plates were stained with a $0.4 \%(\mathrm{w} / \mathrm{v})$ crystal violet solution in $10 \mathrm{~min}$ and washed. Biofilm formation was decided by the $200 \mu \mathrm{l}$ of $33 \%$ glacial acid for $10 \mathrm{~min}$ and measured the value of $\mathrm{OD}_{492}$ with a microplate reader. Each experiment was repeated three times separately. When the value of $\mathrm{A}_{492}$ of $\geq 0.17$, we defined that it was a biofilm positive isolate. The $S$. epidermidis RP62A (ATCC 35984) and the S. epidermidis ATCC12228 were used as positive and negative controls, respectively.

\section{Competition with Staphylococcus aureus}

This assay was experimented according to the previous study [18]. Briefly, each tested S. epidermidis isolate grew overnight in TSB. After incubation at $37^{\circ} \mathrm{C}$, the turbidities of tested S. epidermidis isolates at $600 \mathrm{~nm}$ were measured using a microplate reader. The $\mathrm{OD}_{600}$ value (0.2) of each tested isolate was adjusted to the same concentration of $1.0 \times 10^{5} \mathrm{CFU} / 100 \mu \mathrm{l}$. S. aureus 75 strain (SA75) associated with skin and soft tissue infection was selected as competence object of $S$. epidermidis isolates, and adjusted to the same concentration. Then SA75 and S. epidermidis isolates were simultaneously diluted to 1:200 and added into TSB liquid medium. After a $9 \mathrm{~h}$ incubation at $37^{\circ} \mathrm{C}$, the broth with mixture of $S$. epidermidis and $S$. aureus was diluted to $0.25 \times 10^{-6}$. $100 \mu \mathrm{l}$ of the diluted culture was plated onto blood agar plates and incubated at $37^{\circ} \mathrm{C}$ overnight. The amounts of colonies of S. epidermidis isolate and SA75 on the plate were counted, which was on the basis of SA75 isolate used being beta-haemolytic. The ratio of the numbers of colonies of tested S. epidermidis isolate and SA75 was used for evaluating the competence of $S$. epidermidis isolate with SA75. The experiment was repeated twice.

\section{Statistical analysis}

Statistical software (version 19, IBM SPSS Statistics) was used for all data analyses.

Fisher's exact test or the chi-square test was used to compare positive rates of virulence-associated genes and antimicrobial resistance. T-test was used to compare the competence among different STs. The competition assay were analyzed using Graphpad Prism software (version 7.00, La Jolla, CA, United States), and $p$-value $<0.05$ was considered statistically significant. Statistical significance was achieved with 2 -sided $P$ value of 0.05 .

\section{Results}

Antimicrobial susceptibility characteristics

97(43.49\%) and 63 (59.43\%) clinical and colonized isolates positive for $m e c A$ determined by PCR were identified as MRSE. The prevalence of MRSE among clinical isolates was significantly higher than that among colonized isolates $(P<$ 0.01). The resistance rates of clinical and colonized isolates to antimicrobials tested were listed in Table 1. All tested isolates were susceptible to vancomycin. Only one colonized isolate were resistant to linezolid, while none of those clinical isolates were resistant to linezolid. The resistance rates of clinical isolates to penicillin, tetracycline, ciprofloxacin, chloramphenicol, gentamicin, clindamycin, erythromycin andtrimethoprim-sulfamethoxazole were significantly higher than those among colonized isolates.

\section{Prevalence of mupA and $q a c A / B$}

The positive rates of mup $A$ and $q a c A / B$ among clinical and colonized $S$. epidermidis isolates were 18.38 and $44.84 \%$, and 10.38 and $53.77 \%$, respectively. Although the prevalence of mupA among clinical isolates was higher than that of colonized isolates, there was no difference between two groups. But the prevalence of $q a c A / B$ was reserved.

\section{Prevalence of virulence-associated genes and biofilm formation}

The positive rates of virulence-associated genes among clinical and colonized S. epidermidis isolates were showed in Table 1. IS256 was found among $43.49 \%$ of clinical isolates while only $1.89 \%$ of colonized isolates. In the present study, ACME-acrA was also found both in clinical (52.02\%) and colonized (34.91\%) S. epidermidis isolates. The positive rates of altE and aae among clinical isolates were high to $97.76 \%$, while only 13.21 and $12.26 \%$ among colonized isolates. The positive rates of virulence-associated genes except icaA among $S$. epidermidis clinical isolates were significantly higher than 
Table 1 Antimicrobial resistance profiles and carriage of virulence genes among S. epidermidis clinical and colonized isolates

\begin{tabular}{|c|c|c|c|c|c|}
\hline & \multicolumn{2}{|c|}{$\begin{array}{l}\text { Clinical isolates } \\
(N=223)\end{array}$} & \multicolumn{2}{|c|}{$\begin{array}{l}\text { Colonized isolates } \\
(N=106)\end{array}$} & \multirow[t]{2}{*}{$\begin{array}{l}P \\
\text { value }\end{array}$} \\
\hline & No. & $\%$ & No. & $\%$ & \\
\hline \multicolumn{6}{|l|}{ Antimicrobials } \\
\hline penicillin & 213 & 95.52 & 87 & 82.08 & 0.000 \\
\hline tetracycline & 76 & 34.08 & 19 & 17.92 & 0.004 \\
\hline ciprofloxacin & 66 & 29.60 & 37 & 34.91 & 0.000 \\
\hline chloramphenicol & 78 & 34.98 & 10 & 9.43 & 0.000 \\
\hline gentamicin & 72 & 32.29 & 21 & 19.81 & 0.000 \\
\hline clindamycin & 110 & 49.33 & 36 & 33.96 & 0.000 \\
\hline erythromycin & 184 & 82.51 & 66 & 62.26 & 0.000 \\
\hline $\begin{array}{l}\text { Trimethoprim/ } \\
\text { sulfamethoxazole }\end{array}$ & 99 & 44.39 & 27 & 25.47 & 0.000 \\
\hline linezolid & 0 & 0.00 & 1 & 0.94 & 0.684 \\
\hline vancomycin & 0 & 0.00 & 0 & 0.00 & $N A^{a}$ \\
\hline Biofilm formation & 21 & 9.42 & 19 & 17.92 & \\
\hline \multicolumn{6}{|l|}{ Virulence genes } \\
\hline$i c a A$ & 46 & 20.63 & 24 & 22.64 & 0.202 \\
\hline$a a p$ & 148 & 66.37 & 61 & 57.55 & 0.022 \\
\hline sesl & 39 & 17.49 & 4 & 3.77 & 0.000 \\
\hline $\operatorname{arcA}$ & 112 & 52.02 & 37 & 34.91 & 0.000 \\
\hline $1 S 256$ & 97 & 43.50 & 2 & 1.89 & 0.000 \\
\hline Bhp & 102 & 22.32 & 0 & 0.00 & 0.000 \\
\hline Aae & 443 & 96.94 & 13 & 12.26 & 0.000 \\
\hline GehD & 168 & 36.76 & 7 & 6.60 & 0.000 \\
\hline \multicolumn{6}{|l|}{ Resistance genes } \\
\hline mupA & 41 & 18.38 & 11 & 10.38 & 0.065 \\
\hline$q a c A / B$ & 100 & 44.84 & 57 & 53.77 & 0.058 \\
\hline
\end{tabular}

${ }^{\mathrm{a}} N A$ not analyzed

those among colonized isolates $(P<0.05)$. There were no differences in the positive rates of biofilm formation between clinical isolate and colonized isolates. However, the positive rates of another biofilm-associated gene, aap, among clinical and colonized isolates were relatively high.

\section{Genetic diversity of S. epidermidis clinical and colonized isolates}

A total of 60 STs were identified by MLST among the 329 isolates analyzed (Table 2). The Fig. 1 were showed that eBURST analysis of S.epidermidis using all STs available in the MLST database. Among the 223 clinical isolates, 28 STs were identified, with ST2 the most prevalent ST (28 isolates, 12.56\%), followed by ST466 (20 isolates, 8.97\%). The other STs were included merely
Table 2 Sequence types (ST) distribution among S. epidermidis clinical isolates and colonized isolates

\begin{tabular}{|c|c|c|c|c|c|}
\hline \multirow[t]{2}{*}{ MLST } & \multirow[t]{2}{*}{$\mathrm{CC}$} & \multicolumn{2}{|c|}{$\begin{array}{l}\text { Clinical isolates }(N= \\
223)\end{array}$} & \multicolumn{2}{|c|}{$\begin{array}{l}\text { Colonized isolates }(N= \\
106)\end{array}$} \\
\hline & & No. & $\%$ & No. & $\%$ \\
\hline $\mathrm{ST} 2$ & 2 & 28 & $12.56 \%$ & 1 & $0.94 \%$ \\
\hline ST5 & 2 & 1 & $0.45 \%$ & 0 & $0.00 \%$ \\
\hline ST10 & 2 & 2 & $0.90 \%$ & 0 & $0.00 \%$ \\
\hline ST14 & 2 & 7 & $3.14 \%$ & 5 & $4.72 \%$ \\
\hline ST16 & 2 & 2 & $0.90 \%$ & 0 & $0.00 \%$ \\
\hline ST20 & 2 & 8 & $3.59 \%$ & 14 & $13.21 \%$ \\
\hline ST21 & 2 & 3 & $1.35 \%$ & 0 & $0.00 \%$ \\
\hline ST57 & 2 & 4 & $1.79 \%$ & 2 & $1.89 \%$ \\
\hline ST59 & 2 & 12 & $5.38 \%$ & 3 & $2.83 \%$ \\
\hline ST89 & 2 & 3 & $1.35 \%$ & 0 & $0.00 \%$ \\
\hline ST125 & 2 & 6 & $2.69 \%$ & 1 & $0.94 \%$ \\
\hline ST130 & 2 & 16 & $7.17 \%$ & 19 & $17.92 \%$ \\
\hline ST210 & 2 & 9 & $4.04 \%$ & 1 & $0.94 \%$ \\
\hline ST226 & Singletons & 4 & $1.79 \%$ & 0 & $0.00 \%$ \\
\hline ST227 & 227 & 5 & $2.24 \%$ & 1 & $0.94 \%$ \\
\hline ST234 & 2 & 3 & $1.35 \%$ & 1 & $0.94 \%$ \\
\hline ST235 & 2 & 6 & $2.69 \%$ & 0 & $0.00 \%$ \\
\hline ST248 & 2 & 1 & $0.45 \%$ & 1 & $0.94 \%$ \\
\hline ST262 & Singletons & 4 & $1.79 \%$ & 0 & $0.00 \%$ \\
\hline ST263 & 365 & 5 & $2.24 \%$ & 1 & $0.94 \%$ \\
\hline ST466 & 2 & 20 & $8.97 \%$ & 16 & $15.09 \%$ \\
\hline Non typing & & 13 & $5.83 \%$ & 12 & $11.32 \%$ \\
\hline Other & & 61 & $27.35 \%$ & 28 & $26.42 \%$ \\
\hline Total & & 223 & $100.00 \%$ & 106 & $100.00 \%$ \\
\hline
\end{tabular}

one or several strains. 32 STs were identified among 106 colonized isolates. The major STs were ST130 (19 isolates, 17.92\%), ST466 (16 isolates, 15.09\%) and ST20 (14 isolates, 13.21\%). However, only one colonized isolate belonged to ST2. The other 29 dispersed STs correspond to $53.77 \%$ of the colonized isolates. Moreover, the STs of 13 clinical and 12 colonized isolates were not matched with known STs in online database (http://www.mlst. net), which may be novel STs. Twenty one STs listed in Table 2 were exclusively found among clinical isolates. Six STs including ST32, ST83, ST138, ST141, ST230 and ST325 were exclusively identified among colonized isolates. Twenty-seven STs mainly including ST466, ST130, ST20, ST59 and ST14 were found both clinical and colonized isolates. The prevalent STs accounting for more than 20 clinical isolates, including ST2, ST466, ST59, ST130 and ST20 were designated as major clones.

The eBURST algorithm clustered all of the 60 STs isolated of clinical isolates into clonal complex 2 (CC2) (166/ 


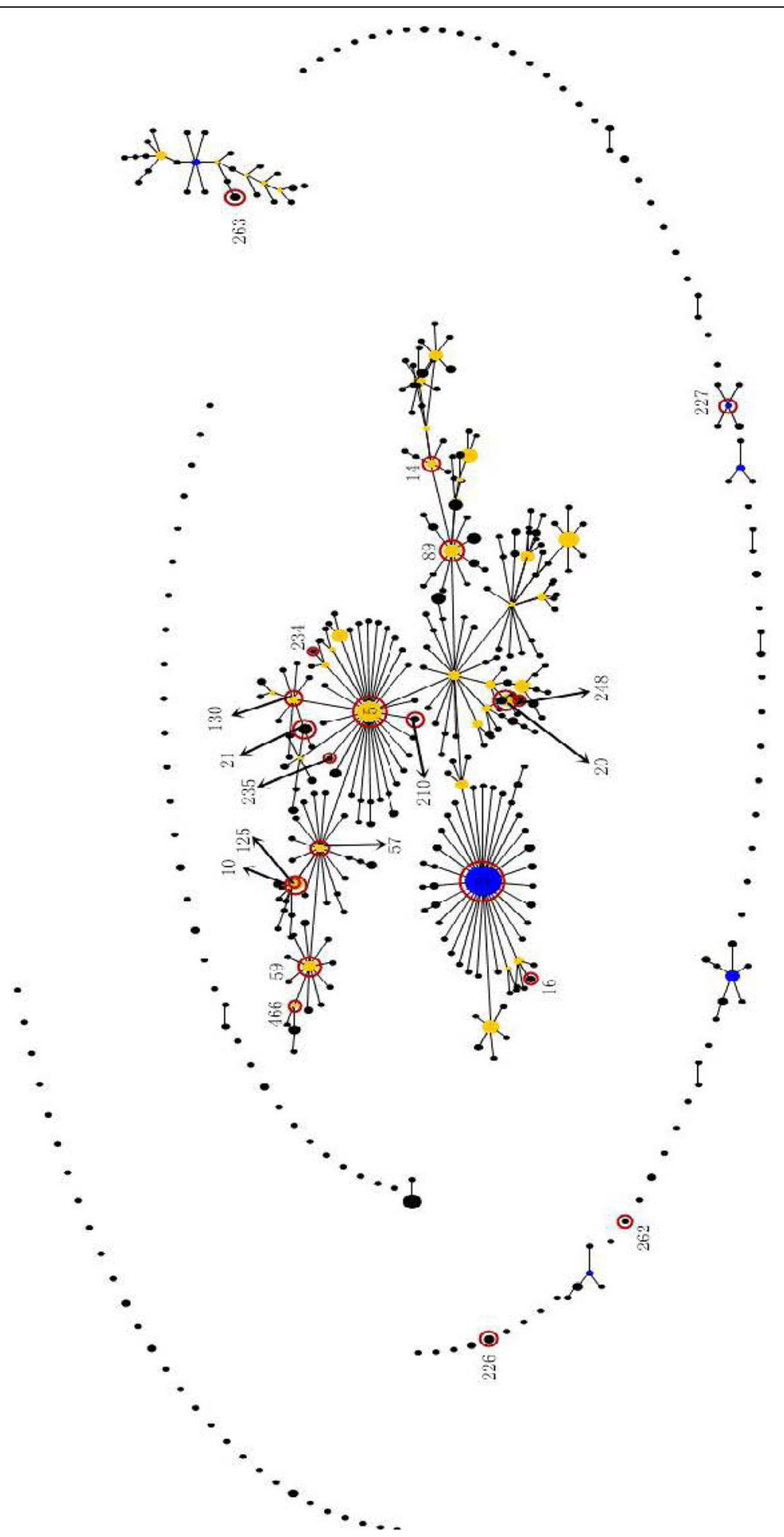

Fig. 1 eBURST analysis of S.epidermidis using all STs available in the MLST database. ST2 was the founder of S. epidermidis in MLST database. ST5, the single locus variant (SLV) of ST2, was the founder of clinical isolates, while ST85, also the SLV of ST2, was the founder of colonized isolates

223; 74.40\%), 7 minor CCs (CC365, CC193, CC227, CC171, CC33, CC326, and CC66, 16/223, 7.17\%), and 29 singletons. Among the 106 colonized isolates, 5 CCs were identified, including CC2 (77/106; 72.64\%), CC365 (4 isolates), CC193 (1 isolates), CC227 (1 isolates) and CC66 (1 isolates) as shown in Fig. 2b. ST2 was the founder of $S$. 

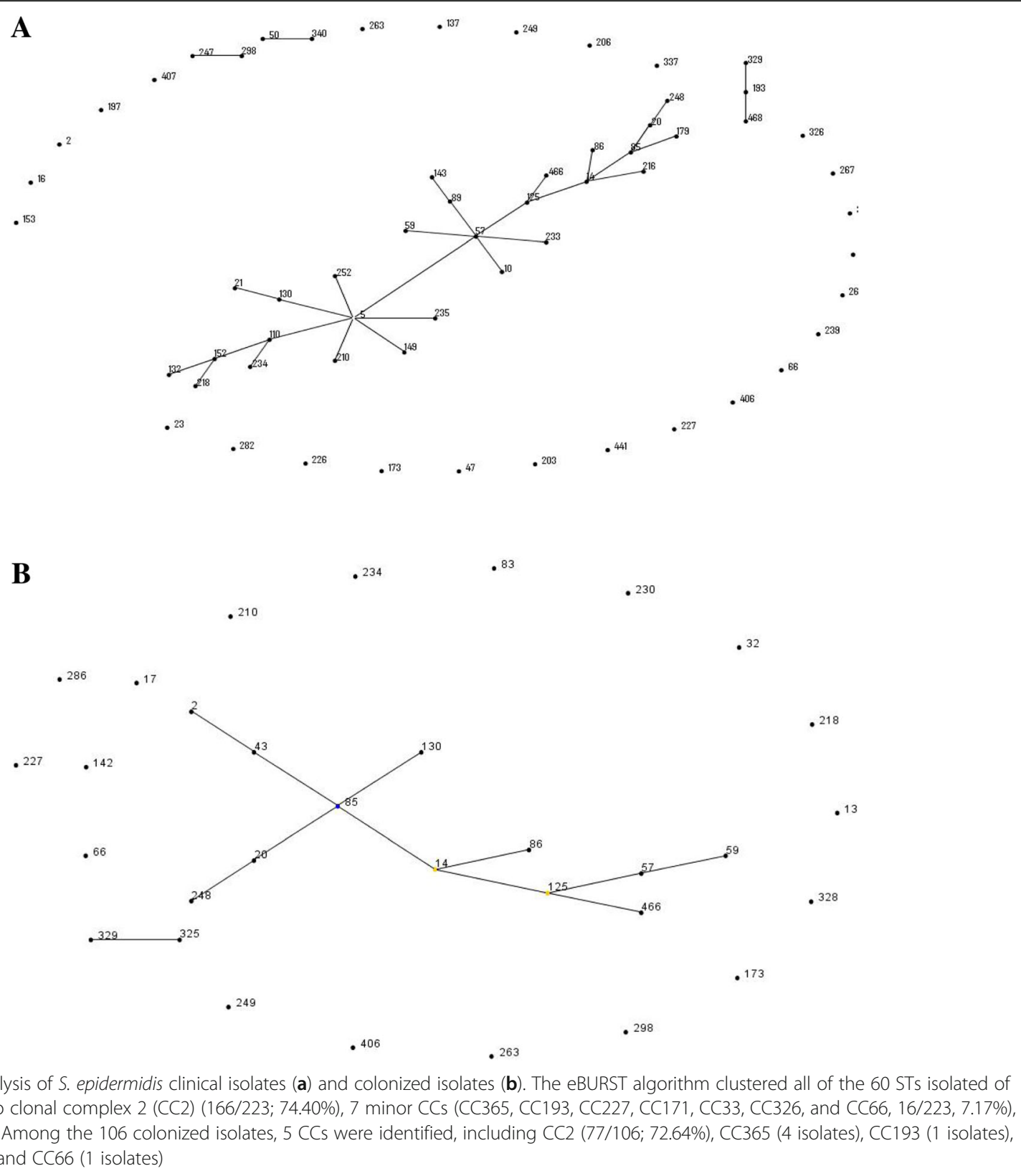

Fig. 2 eBURST analysis of S. epidermidis clinical isolates (a) and colonized isolates (b). The eBURST algorithm clustered all of the 60 STs isolated of clinical isolates into clonal complex 2 (CC2) (166/223; 74.40\%), 7 minor CCs (CC365, CC193, CC227, CC171, CC33, CC326, and CC66, 16/223, 7.17\%), and 29 singletons. Among the 106 colonized isolates, 5 CCs were identified, including CC2 (77/106; 72.64\%), CC365 (4 isolates), CC193 (1 isolates), CC227 (1 isolates) and CC66 (1 isolates)

epidermidis in MLST database. In the present study, ST5, the single locus variant (SLV) of ST2, was the founder of clinical isolates, while ST85, also the SLV of ST2, was the founder of colonized isolates. Although 60 different STs were identified among S. epidermidis isolates analyzed, the great majority of the isolates $(73.8 \%, 243 / 329)$ belonged to a single clone complex, CC2.

\section{Comparison of characteristics of ST466 clone with other major clones among clinical isolates}

The characteristics of 5 major clones were showed in Table 3. a) Antimicrobial resistance profiles and prevalence of mupA and $q a c A / B$

Interestingly, the prevalence of MRSE among these 5 major clones was high to more than 95\%, especially ST59 and ST20 clones with MRSE prevalence of $100 \%$. The resistance rates of ST2 clone to ciprofloxacin, gentamicin, clindamycin, erythromycin and trimethoprim-sulfamethoxazole were more than $65 \%$, which were higher than other clones, but with the lowest resistance rate of tetracycline(10.71\%). In contrast to ST2 clone, the second prevalent clone, ST466, had the highest resistance rate to tetracycline $(50.00 \%)$ relative to other clones. The resistance rates of ST466 clone to 
Table 3 The antimicrobial resistance rates, positive rates of virulence genes and of resistance genes among S.epidermidis clinical isolates with major ST types (\%)

\begin{tabular}{|c|c|c|c|c|c|}
\hline & ST466 $(N=20)$ & $\mathrm{ST} 2(N=28)$ & ST130 $(N=16)$ & ST20 $(N=8)$ & ST59 $(N=12)$ \\
\hline \multicolumn{6}{|l|}{ Antimicrobials } \\
\hline penicillin & $95(19)$ & $96.43(27)$ & $100.00(16)$ & 100.00(8) & $91(11)$ \\
\hline tetracycline & $50.00(10)$ & 10.71(3) & $25.00(4)$ & $50.00(4)$ & $33.33(4)$ \\
\hline ciprofloxacin & $15.00(3)$ & $82.14(23)$ & $25.00(4)$ & $50.00(4)$ & 33.33(4) \\
\hline chloramphenicol & $30.00(6)$ & $25.00(7)$ & $12.50(2)$ & $12.50(1)$ & $0.00(0)$ \\
\hline gentamicin & $20.00(4)$ & $82.14(23)$ & $25.00(4)$ & $0.00(0)$ & $33.33(4)$ \\
\hline clindamycin & $30.00(6)$ & 67.86(19) & $50.00(8)$ & $25.00(2)$ & $41.67(5)$ \\
\hline erythromycin & $80.00(16)$ & $82.14(23)$ & $68.75(11)$ & $87.50(7)$ & 83.33(10) \\
\hline$S X T^{a}$ & $60.00(12)$ & $71.43(20)$ & $100(16)$ & $0.00(0)$ & $41.67(5)$ \\
\hline linezolid & $0.00(0)$ & $0.00(0)$ & $0.00(0)$ & $0.00(0)$ & $0.00(0)$ \\
\hline vacomycin & $0.00(0)$ & $0.00(0)$ & $0.00(0)$ & $0.00(0)$ & $0.00(0)$ \\
\hline Biofilm formation & $5.00(1)$ & $10.71(3)$ & $0.00(0)$ & $75.00(6)$ & $0.00(0)$ \\
\hline \multicolumn{6}{|l|}{ Virulence genes } \\
\hline$i c a A$ & $0.00(0)$ & $85.71(24)$ & $0.00(0)$ & $62.50(5)$ & $0.00(0)$ \\
\hline aap & $90.00(18)$ & $96.43(27)$ & $50.00(8)$ & $50.00(8)$ & $91.67(11)$ \\
\hline sesl & $0.00(0)$ & $96.43(27)$ & $0.00(0)$ & $0.00(0)$ & $25.00(3)$ \\
\hline ACME-arcA & $65.00(13)$ & $39.29(11)$ & $87.50(14)$ & $25.00(2)$ & $41.67(5)$ \\
\hline IS256 & $15.00(3)$ & $82.14(23)$ & $43.75(7)$ & $25.00(2)$ & 33.33(4) \\
\hline bhp & $30.00(6)$ & $10.71(3)$ & $0.00(0)$ & $0.00(0)$ & $50.00(6)$ \\
\hline aae & $100.00(20)$ & $92.86(26)$ & $100.00(16)$ & 100.00(8) & $100.00(12)$ \\
\hline alte & $100.00(20)$ & $96.43(27)$ & $100.00(16)$ & 100.00(8) & $100.00(12)$ \\
\hline gehD & $25.00(5)$ & 67.86(19) & $12.50(2)$ & $62.50(5)$ & $33.33(4)$ \\
\hline \multicolumn{6}{|l|}{ Resistance genes } \\
\hline mupA & $0.00(0)$ & $39.29(11)$ & $12.50(2)$ & $12.50(1)$ & $8.33(1)$ \\
\hline$q a c A / B$ & $65.00(13)$ & 67.86(19) & $56.25(9)$ & $62.50(5)$ & $58.33(7)$ \\
\hline
\end{tabular}

antimicrobials tested were similar to ST59 clone except chloramphenicol. The resistance rates of ST20 clone to trimethoprim-sulfamethoxazole, gentamicin, clindamycin and chloramphenical were lower than other clones, especially to trimethoprim-sulfamethoxazole $(0.0 \%)$. The positive rates of mup $A$ and $q a c A / B$ among ST2 clone were higher than other 4 clones. The prevalence of $q a c A / B$ among ST466 clone was near to that of ST2 and 59 clones, with a relatively high prevalence of $q a c A / B$.

b) Biofilm formation and carriage of virulenceassociated genes

Although the prevalence of biofilm formation among clinical isolates was only $9.42 \%$, that among ST20 clone was high to $75.00 \%$, followed by ST2 clone (10.71\%), which was associated with more than $60 \%$ of ST2 and ST20 clones with icaA. Conversely, among clinical isolates all ST466 clone except one isolate and ST59 clone were negative for icaA and biofilm formation. In the present study, the prevalence of IS256 among ST2 clone was $82.14 \%$, which was significantly higher than other clones. The highest prevalence of colonizationassociated element, ACME, was found among ST130 clone $(87.50,14 / 16)$, followed by ST466 clone $(65.00 \%$, 13/20). The prevalence of sesI among ST2 clone was high to $96.43 \%(27 / 28)$. However, the positive rates of sesI among other 4 clones were low, especially no ST466 clone positive for this gene. Other than ST130 clone with relatively low aap prevalence $(50.00 \%, 8 / 16)$, the positive rates of aap for ST2 $(96.43 \%, 27 / 28)$, ST466 $(90.00 \%, 18 / 20)$, ST59 $(91.67 \%, 11 / 12)$ and ST20 $(100.00 \%, 8 / 8)$ clones were over $90 \%$. The positive rates of altE and aae among 5 prevalent clones were high to more than 90\%, with all ST20, ST130 and ST59 clones positive for these two surface-associated autolysin/adhesion genes. The pattern of virulence-associated genes among ST466 was similar to ST59 except sesI. Compared with the predominant ST2 clone, ST466 clone had higher positive rates of ACME-arcA, altE, aae and bhp. 


\section{c) Competence with S. aureus of ST466}

The competence of ST466 clone with S. aureus were significantly stronger than ST2 and ST130 clones (Fig. 3) $(P<0.05)$.Although the competence of ST466 clone with S. aureus was higher than ST20 and ST59 clones, there were no differences among them, especially ST59 with similar competence relative to ST466.

\section{Discussion}

Both similarities and differences were detected in the genotypes, virulence-associated genes, antimicrobial susceptibility characteristics, ability of biofilm formation, STs and CC amongst S.epidermidis from clinical isolates and colonized isolates. Previous study showed that S. epidermidis isolates from patients with catheter-related bloodstream infection were significantly more resistant to most of non-beta lactam drugs than the S. epidermidis isolates collected from healthcare workers [19]. However, the resistance rates to clindamycin among S. epidermidis isolates were different from country to country. In most countries, clindamycin resistance rates were relatively high (>40\%) [20-24]. The resistance rates of S. epidermidis clinical isolates to clindamycin in Northern of Jordan (57.4\%) [25] and Belgian (75\%) [19] were relatively high. In contrast, a previous report from UK showed that resistance rate of $S$. epidermidis isolates from 10 hospitals to clindamycin was low to $13 \%$ (82/633) [26].

Surface-active antiseptics such as chlorhexidine and mupirocin treatment were comprehensive strategy in reducing S. aureus and S. epidermidis colonization and infection in the hospital were strongly recommended as decolonization agents. Emergence of resistance to antiseptics and mupirocin will threaten the hospital infection control. In the present study, a high prevalence of

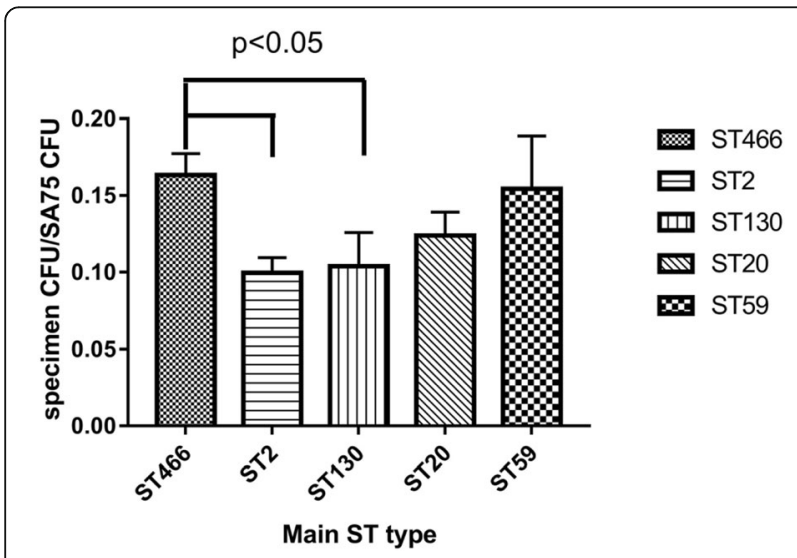

Fig. 3 The competence of ST466 clone with S. aureus (SA75) were significantly stronger than ST2 and ST130 clones (Fig. 3) $(P<0.05)$. Although the competence of ST466 clone with S. aureus was higher than ST20 and ST59 clones, there were no differences among them, especially ST59 with similar competence relative to ST466 mup $A$ and $q a c A / B$ was found among S. epidermidis clinical and colonized isolates, which should be of concern.

Numerous infections caused by S. epidermidis involves biofilm formation, which is the most important factor involved in its pathogenesis [27].Some studies have shown that commensal S. epidermidis populations differ from clinical isolates in the frequency of the carriage of virulence genes such as icaA involved in biofilm formation [28-32]. Although no virulence determinants can be used to clearly distinguish $S$. epidermidis clinical isolates from colonized isolates [33, 34], IS256 was found different between clinical isolates and colonized isolates. Previous study indicated that sesI may be a marker of S. epidermidis invasive capacity, because it was not found among healthy individuals, but was found in clinical isolates (approximately 50\%) associated invasive infections [12]. In the present study, although the sesI prevalence in clinical isolates was lower than previous studies [12, 35], it was in accordance with the findings that sesI is more frequently found among clinical isolates than that in colonized isolates. A novel genomic island named arginine catabolic mobile element (ACME) may increase the colonized capacity of $S$. epidermidis to the human skin, mucosal surfaces and in-dwelling medical devices [36]. ACME was found in both clinical and colonized S. epidermidis isolates, indicating that ACME played a more important role in colonization than in virulence [37]. In the present study, the percentage of ACME-acrA was lower than previous reports $(>70 \%)[7,37]$, but similar to the previous study that indicating the percentage of ACME-acrA of $S$. epidermidis isolates from a widespread geographical origin was $51 \%[38]$.

A surface-associated autolysin/adhesin, AtlE, from S. epidermidis mediates initial adherence of bacterial cells to the polymer surface [39]. Interestingly, all the isolates harboring altE except one colonized isolate were simultaneously positive for aae encoding another multifunctional autolysin/adhesin with bacteriolytic activity that binds to fibrinogen, vitronectin, and fibronectin. Bhp, a protein homologous to Bap was assumed to promote biofilm formation [1]. Surprisingly, $b h p$ was exclusively found among clinical isolates, indicating that there was an association between carriage of $b h p$ and pathogenicity of S. epidermidis.

Different results of ST type distribution indicated that the $S$. epidermidis tested in the present study showed considerable genetic diversity. The comparison of the isolated from the patients and healthy volunteers showed that two groups had a high level of genetic diversity, but the clinical isolates were even more diverse than the colonized isolates. Characteristics of highly diversity of $S$. epidermidis isolates were also observed in other studies [29, 40]. It was speculated that this genetic diversity 
might be caused by the need of the $S$. epidermidis to adapt to the different environments in the hospital and community settings [6]. ST2 has usually been the most prevalent ST observed in previous epidemiological studies among S.epidermidis strains worldwide, especially in BSIs and catheter-related infections [6, 41]. Furthermore, ST2 has been associated with many of the currently known virulence factors, such as biofilm production and antibiotic resistance [7, 41]. In the present study, ST2 was the most dominant type in clinical isolates, which was similar to previous studies [6, 41-44]. Interestingly, ST466 was the second prevalent ST among clinical isolates and colonized isolates. Up to now, only a report from Shanghai of China found that a minority of S. epidermidis nasal isolates from healthcare staff at a teaching hospital belonged to ST466 [7]. In addition to China, other countries did not found ST466. The spread of ST466 clone between clinical and colonized isolates indicated that this clone may be become the main cause of infections by $S$. epidermidis in China, which should be of concern.

In our study, the high prevalence of $\mathrm{CC} 2$ has been found both in clinical isolates and colonized isolates, indicating that $\mathrm{CC} 2$ possibly adapted to different environment by recombination or mutation. A report from China showed that $91.7 \%$ (297/ 324) of S. epidermidis from the community and hospital environments belonged to CC2 [7]. Our data support the evidence that overwhelming majority of $S$. epidermidis isolates from China belong to CC2 regardless of clinical isolates or colonized isolates. $\mathrm{CC} 2$ comprised of $74 \%$ of the S. epidermidis isolates from 17 national centers between 1996 and 2001 [6]. The majority (62/71, 87.3\%) of S. epidermidis clinical isolates from U.S. hospitals belonged to CC2 [45]. Although molecular typing of S. epidermidis isolates from diverse geographic or clinical origins by MLST has shown considerable diversity, as shown in Fig. 2, $\mathrm{CC} 2$ is an overwhelming clonal lineage worldwide.

Our study showed that different clones were associated with characteristic non- $\beta$-lactam antimicrobial resistance and resistance genes patterns, suggesting that the clinical selection of antimicrobials based on typing molecular is help for the treatment of S. epidermidis infections and decolonization of S. epidermidis. Our data also suggested that high prevalence of MRSE, multiple antibiotic resistance and genes responsible for resistance to surface-active antiseptics and mupirocin used for decolonization of Staphylococci, contributes to the spread of the prevalent $S$. epidermidis clones in hospital environment, including a novel and rarely encountered clone, ST466.

A report from China showed that majority of the predominant clinical MRSE clone ST2 isolates were positive for biofilm-related genes IS256 (81.0\%) and icaA (75.0\%) [7]. The high prevalence of virulence-associated genes and resistance to antimicrobials in ST2 of S. epidermidis isolates indicating that ST2 were associated with spread. In the present study, majority of sesI-positive $S$. epidermidis clinical isolates belonged to ST2, while few sesInegative clinical isolates belonged to this clone, indicating that there was strong association between carriage of sesI and ST2 clone. While percentage of sesI and icaA in ST466 was $0.0 \%$, we speculated that the prevalence of ST466 may not depend on formation of biofilm.The carriage of high prevalence of multiple virulence-associated genes including ACME-arcA, altE, aae, aap and bhp maybe contribute to the spread of ST466 clone. As ST466 was a single locus variant of ST59, the antimicrobial resistance profiles and the patterns of resistance genes and virulence-associated genes for ST466 clone were similar to those for ST59 clone.

As cross-interfering quorum-sensing pheromones were discovered in staphylococci, quorum-sensing crossinhibition was believe to be the source for a potential interference between S. epidermidis and S. aureus [34]. Colonization with $S$. epidermidis can prevent overgrowth of the more aggressive $S$. aureus [46]. S. epidermidis strains were reported by Iwase et al. to can express a certain secreted protease Esp which prevented nasal colonization with $S$. aureus [47]. The underlying mechanism that ST466 clone had more competence with $S$. aureus relative to other 4 major clones was not known and should be further investigated.

\section{Conclusions}

Taken together, a high-level of genetic diversity was found between clinical and colonized S. epidermidis isolates, with clinical $S$. epidermidis isolates with more resistance to clinically often used antimicrobial agents and carriage of virulence-associated genes. A novel ST466 clone with distinct and similar characteristics relative to other prevalent clones, emerging as a prevalent clone in China, should be of major concern.

\section{Additional files}

Additional file 1: Drug susceptibility results of 223 strains of clinical S. epidermidis". MIC values of 10 drug susceptibility results of 223 clinical S. epidermidis. (PDF 194 kb)

Additional file 2: Drug susceptibility results of 106 strains of colonized S. epidermidis". MIC values of 10 drug susceptibility results of 106 colonized S. epidermidis. (PDF $112 \mathrm{~kb}$ )

\section{Abbreviations}

ACME: Arginine catabolic mobile element; CC2: Clone complex 2; CLSI: Clinical and Laboratory Standards Institute; CoNS: Coagulase-negative Staphylococci; MLST: Multi-locus sequence typing; MRSE: Methicillin-resistant S. epidermidis; PIA: Polysaccharideinter cellular adhesion; PNAG: Polymericnacetyl-glucosamine; SCCmec: Staphylococcal chromosomal cassette mec 


\section{Acknowledgements}

The authors are grateful to the First Affiliated Hospital of Wenzhou Medical University.

\section{Authors' contributions}

$Y G, Y D, L L, X S, J D, Z H$ and $Y J$ performed the laboratory measurements. ZC and FY made substantial contributions to conception and design. YG and FY revised the manuscript critically for important intellectual content. FY drafted the manuscript. All authors read and approved the final manuscript.

\section{Author's information}

Member of Shanghai Pulmonary Hospital: Yinjuan Guo, and Fangyou Yu. Member of the First Affiliated Hospital of Wenzhou Medical University: Li Liu, Xiaofei Shen, Zhihao Hao, Jingjing Duan, Ye Jin, Zengqiang Chen.

Member of Hunan Provincial People's Hospital: Yu Ding.

\section{Funding}

This study was supported by grants from Natural Science fund of China (81472011). It supported the each section of this study, including design of the study and collection, analysis, and interpretation of data and in writing the manuscript.

\section{Availability of data and materials}

The datasets used during the current study are available from the corresponding author upon reasonable request. Most of the data is included in this published article [and Additional files 1 and 2].

\section{Ethics approval and consent to participate}

The Ethics Committee of the first Affiliated Hospital of Wenzhou Medical University exempted this study from review because the present study focused on bacteria.

\section{Consent for publication}

Not applicable.

\section{Competing interests}

The authors declare they have no competing interests.

\section{Author details}

'Department of Laboratory Medicine, Shanghai Pulmonary Hospital, Tongji University School of Medicine, Shanghai 200082, China. ${ }^{2}$ Department of Laboratory Medicine, Hunan Provincial People's Hospital, Changsha 410000, China. ${ }^{3}$ Department of Laboratory Medicine, the First Affiliated Hospital of Wenzhou Medical University, Wenzhou 325000, China.

\section{Received: 16 July 2018 Accepted: 20 June 2019}

Published online: 09 July 2019

\section{References}

1. Becker K, Heilmann C, Peters G. Coagulase-negative staphylococci. Clin Microbiol Rev. 2014;27(4):870-926.

2. Otto M. Virulence factors of the coagulase-negative staphylococci. Front Biosci. 2004;9:841-63.

3. Onishi M, Urushibara N, Kawaguchiya M, Ghosh S, Shinagawa M, Watanabe $\mathrm{N}$, Kobayashi N. Prevalence and genetic diversity of arginine catabolic mobile element (ACME) in clinical isolates of coagulase-negative staphylococci: identification of ACME type I variants in Staphylococcus epidermidis. Infect Genet Evol. 2013;20:381-8.

4. Martins A, Cunha Mde L. Methicillin resistance in Staphylococcus aureus and coagulase-negative staphylococci: epidemiological and molecular aspects. Microbiol Immunol. 2007;51(9):787-95.

5. Widerstrom M, Wistrom J, Sjostedt A, Monsen T. Coagulase-negative staphylococci: update on the molecular epidemiology and clinical presentation, with a focus on Staphylococcus epidermidis and Staphylococcus saprophyticus. Eur J Clin Microbiol Infect Dis. 2012;31(1):7-20.

6. Miragaia M, Thomas JC, Couto I, Enright MC, de Lencastre $\mathrm{H}$. Inferring a population structure for Staphylococcus epidermidis from multilocus sequence typing data. J Bacteriol. 2007;189(6):2540-52.

7. Du X, Zhu Y, Song Y, Li T, Luo T, Sun G, Yang C, Cao C, Lu Y, Li M. Molecular analysis of Staphylococcus epidermidis strains isolated from community and hospital environments in China. PLoS One. 2013;8(5):e62742.
8. CLSI. Performance standards for antimicrobial susceptibility testing, 24th informational supplement (M100-S24). Wayne: Clinical and Laboratory Standards Institute; 2014

9. Enright MC, Day NP, Davies CE, Peacock SJ, Spratt BG. Multilocus sequence typing for characterization of methicillin-resistant and methicillin-susceptible clones of Staphylococcus aureus. J Clin Microbiol. 2000;38(3):1008-15.

10. Murakami K, Minamide W, Wada K, Nakamura E, Teraoka H, Watanabe S. Identification of methicillin-resistant strains of staphylococci by polymerase chain reaction. J Clin Microbiol. 1991;29(10):2240-4.

11. Catalanotti P, Lanza M, Del Prete A, Lucido M, Catania MR, Galle F, Boggia D, Perfetto B, Rossano F. Slime-producing Staphylococcus epidermidis and S. aureus in acute bacterial conjunctivitis in soft contact lens wearers. New Microbiol. 2005;28(4):345-54

12. Soderquist $B$, Andersson M, Nilsson M, Nilsdotter-Augustinsson A, Persson L, Friberg O, Jacobsson S. Staphylococcus epidermidis surface protein I (Sesl): a marker of the invasive capacity of S. epidermidis? J Med Microbiol. 2009;58(Pt 10):1395-7.

13. Cala C, Amodio E, Di Carlo E, Virruso R, Fasciana T, Giammanco A. Biofilm production in Staphylococcus epidermidis strains, isolated from the skin of hospitalized patients: genetic and phenotypic characteristics. New Microbiol. 2015;38(4):521-9.

14. Heilmann C, Thumm G, Chhatwal GS, Hartleib J, Uekotter A, Peters G. Identification and characterization of a novel autolysin (Aae) with adhesive properties from Staphylococcus epidermidis. Microbiology. 2003;149(Pt 10):2769-78.

15. Bowden MG, Visai L, Longshaw CM, Holland KT, Speziale P, Hook M. Is the GehD lipase from Staphylococcus epidermidis a collagen binding adhesin? J Biol Chem. 2002;277(45):43017-23.

16. Li T, Song Y, Zhu Y, Du X, Li M. Current status of Staphylococcus aureus infection in a central teaching hospital in Shanghai, China. BMC Microbiol. 2013;13:153.

17. O'Neill E, Pozzi C, Houston P, Humphreys H, Robinson DA, Loughman A, Foster TJ, O'Gara JP. A novel Staphylococcus aureus biofilm phenotype mediated by the fibronectin-binding proteins, FnBPA and FnBPB. J Bacteriol. 2008;190(11):3835-50.

18. Zipperer A, Konnerth MC, Laux C, Berscheid A, Janek D, Weidenmaier C, Burian M, Schilling NA, Slavetinsky C, Marschal M, et al. Human commensals producing a novel antibiotic impair pathogen colonization. Nature. 2016;535(7613):511-6.

19. Cherifi S, Byl B, Deplano A, Nagant C, Nonhoff C, Denis O, Hallin M. Genetic characteristics and antimicrobial resistance of Staphylococcus epidermidis isolates from patients with catheter-related bloodstream infections and from colonized healthcare workers in a Belgian hospital. Ann Clin Microbiol Antimicrob. 2014;13:20.

20. Said KB, Al-Jarbou AN, Alrouji M, Al-Harbi HO. Surveillance of antimicrobial resistance among clinical isolates recovered from a tertiary care hospital in Al Qassim, Saudi Arabia. Int J Health Sci. 2014;8(1):3-12.

21. Knauer A, Fladerer P, Strempfl C, Krause R, Wenisch C. Effect of hospitalization and antimicrobial therapy on antimicrobial resistance of colonizing Staphylococcus epidermidis. Wien Klin Wochenschr. 2004;116(14):489-94.

22. Soroush S, Jabalameli F, Taherikalani M, Amirmozafari N, Imani Fooladi AA, Asadollahi K, Beigverdi R, Emaneini M. Investigation of biofilm formation ability, antimicrobial resistance and the staphylococcal cassette chromosome mec patterns of methicillin resistant Staphylococcus epidermidis with different sequence types isolated from children. Microb Pathog. 2016;93:126-30.

23. Ahmed MM, Bahlas S. Bacteriological profile and antimicrobial resistance patterns of clinical bacterial isolates in a university hospital. Travel Med Infect Dis. 2009;7(4):235-8.

24. Nakase K, Nakaminami H, Takenaka Y, Hayashi N, Kawashima M, Noguchi N. Relationship between the severity of acne vulgaris and antimicrobial resistance of bacteria isolated from acne lesions in a hospital in Japan. $J$ Med Microbiol. 2014;63(Pt 5):721-8.

25. Al Tayyar IA, Al-Zoubi MS, Hussein E, Khudairat S, Sarosiekf K. Prevalence and antimicrobial susceptibility pattern of coagulase-negative staphylococci (CoNS) isolated from clinical specimens in northern of Jordan. Iran J Microbiol. 2015;7(6):294-301.

26. Andrews J, Ashby J, Jevons G, Marshall T, Lines N, Wise R. A comparison of antimicrobial resistance rates in gram-positive pathogens isolated in the UK from October 1996 to January 1997 and October 1997 to January 1998. J Antimicrob Chemother. 2000;45(3):285-93.

27. Fey PD, Olson ME. Current concepts in biofilm formation of Staphylococcus epidermidis. Future Microbiol. 2010;5(6):917-33. 
28. Rohde H, Burandt EC, Siemssen N, Frommelt L, Burdelski C, Wurster S, Scherpe S, Davies AP, Harris LG, Horstkotte MA, et al. Polysaccharide intercellular adhesin or protein factors in biofilm accumulation of Staphylococcus epidermidis and Staphylococcus aureus isolated from prosthetic hip and knee joint infections. Biomaterials. 2007;28(9):1711-20.

29. Galdbart JO, Morvan A, Desplaces N, el Solh N. Phenotypic and genomic variation among Staphylococcus epidermidis strains infecting joint prostheses. J Clin Microbiol. 1999;37(5):1306-12.

30. Klingenberg C, Ronnestad A, Anderson AS, Abrahamsen TG, Zorman J, Villaruz A, Flaegstad T, Otto M, Sollid JE. Persistent strains of coagulasenegative staphylococci in a neonatal intensive care unit: virulence factors and invasiveness. Clin Microbiol Infect. 2007;13(11):1100-11.

31. Novick RP. Autoinduction and signal transduction in the regulation of staphylococcal virulence. Mol Microbiol. 2003;48(6):1429-49.

32. Schommer NN, Christner M, Hentschke M, Ruckdeschel K, Aepfelbacher M, Rohde H. Staphylococcus epidermidis uses distinct mechanisms of biofilm formation to interfere with phagocytosis and activation of mouse macrophage-like cells 774A.1. Infect Immun. 2011;79(6):2267-76.

33. Kozitskaya S, Cho SH, Dietrich K, Marre R, Naber K, Ziebuhr W. The bacterial insertion sequence element IS256 occurs preferentially in nosocomial Staphylococcus epidermidis isolates: association with biofilm formation and resistance to aminoglycosides. Infect Immun. 2004;72(2):1210-5.

34. Otto M. Molecular basis of Staphylococcus epidermidis infections. Semin Immunopathol. 2012;34(2):201-14.

35. Bowden MG, Chen W, Singvall J, Xu Y, Peacock SJ, Valtulina V, Speziale P, Hook $M$. Identification and preliminary characterization of cell-wall-anchored proteins of Staphylococcus epidermidis. Microbiology. 2005;151(Pt 5):1453-64.

36. Barbier F, Lebeaux D, Hernandez D, Delannoy AS, Caro V, Francois P, Schrenzel J, Ruppe E, Gaillard K, Wolff M, et al. High prevalence of the arginine catabolic mobile element in carriage isolates of methicillin-resistant Staphylococcus epidermidis. J Antimicrob Chemother. 2011;66(1):29-36.

37. Cherifi S, Byl B, Deplano A, Nonhoff C, Denis O, Hallin M. Comparative epidemiology of Staphylococcus epidermidis isolates from patients with catheter-related bacteremia and from healthy volunteers. J Clin Microbiol. 2013;51(5):1541-7.

38. Miragaia M, de Lencastre $\mathrm{H}$, Perdreau-Remington $\mathrm{F}$, Chambers HF, Higashi J, Sullam PM, Lin J, Wong KI, King KA, Otto M, et al. Genetic diversity of arginine catabolic mobile element in Staphylococcus epidermidis. PLoS One. 2009;4(11):e7722.

39. Heilmann C, Hussain M, Peters G, Gotz F. Evidence for autolysin-mediated primary attachment of Staphylococcus epidermidis to a polystyrene surface. Mol Microbiol. 1997;24(5):1013-24.

40. Miragaia M, Couto I, Pereira SF, Kristinsson KG, Westh H, Jarlov JO, Carrico J, Almeida J, Santos-Sanches I, de Lencastre H. Molecular characterization of methicillin-resistant Staphylococcus epidermidis clones: evidence of geographic dissemination. J Clin Microbiol. 2002;40(2):430-8.

41. Li M, Wang X, Gao Q, Lu Y. Molecular characterization of Staphylococcus epidermidis strains isolated from a teaching hospital in Shanghai, China. J Med Microbiol. 2009;58(Pt 4):456-61.

42. Miragaia M, Carrico JA, Thomas JC, Couto I, Enright MC, de Lencastre H. Comparison of molecular typing methods for characterization of Staphylococcus epidermidis: proposal for clone definition. J Clin Microbiol. 2008;46(1):118-29

43. Ibrahem S, Salmenlinna S, Lyytikainen O, Vaara M, Vuopio-Varkila J. Molecular characterization of methicillin-resistant Staphylococcus epidermidis strains from bacteraemic patients. Clin Microbiol Infection. 2008;14(11):1020-7.

44. Widerstrom M, Monsen T, Karlsson C, Edebro H, Johansson A, Wistrom J. Clonality among multidrug-resistant hospital-associated Staphylococcus epidermidis in northern Europe. Scand J Infect Dis. 2009;41(9):642-9.

45. Mendes RE, Deshpande LM, Costello AJ, Farrell DJ. Molecular epidemiology of Staphylococcus epidermidis clinical isolates from U.S. hospitals. Antimicrob Agents Chemother. 2012;56(9):4656-61.

46. von Eiff C, Becker K, Machka K, Stammer H, Peters G. Nasal carriage as a source of Staphylococcus aureus bacteremia. Study Group. N Eng J Med. 2001;344(1):11-6.

47. Iwase T, Uehara Y, Shinji H, Tajima A, Seo H, Takada K, Agata T, Mizunoe Y. Staphylococcus epidermidis Esp inhibits Staphylococcus aureus biofilm formation and nasal colonization. Nature. 2010;465(7296):346-9.

\section{Publisher's Note}

Springer Nature remains neutral with regard to jurisdictional claims in published maps and institutional affiliations.

\section{Ready to submit your research? Choose BMC and benefit from:}

- fast, convenient online submission

- thorough peer review by experienced researchers in your field

- rapid publication on acceptance

- support for research data, including large and complex data types

- gold Open Access which fosters wider collaboration and increased citations

- maximum visibility for your research: over $100 \mathrm{M}$ website views per year

At BMC, research is always in progress.

Learn more biomedcentral.com/submissions 